\title{
La geodinamica al servizio delle grandi dighe
}

\author{
P. CALOT
}

\author{
Ricevuto il 10 Giugno 1964
}

\begin{abstract}
Riassuxto. - Si richiama, ancora nna volta, l'attenzione sull'interesse (a volte fondamentale, anche ai fini pratici) della costante osservazione delle variazioni del campo elastico, nelle zone in cui un mezzo solido è sottoposto a continue variazioni di tensione, commune determinate. L'azione di tormento, associata alle variazioni di tensione, si traduce in un decadimento del modulo elastico, testimoniato dal pin o meno sensibile diminuire della velocita di propagazione delle onde elastiche. L'osservazione continua del campo elastico in una zona sismica, combinata con la contemporanea osservazione della variazione della verticale apparente, puo costituire una valida via d'approcio nel problema della previsione dei terremoti: a questo proposito, nella zona di Tolmezzo, alcune scosse sismiche sono state sorprese nella loro fase di preparazione. Nell'ambito delle grandi dighe, simili indagini, sistematicamente perseguite, possono condurre - come di fatto hanno conflotto - ad indivirluare una locale microsismiciti, naturale o provocata, che puo riuscire esiziale - nelle sue conseguenze - per l'incolumitit delle popolazioni a valle dei grandi sbarramenti. I tragici eventi. verificatisi un po' dovunque negli ultimi anni, suggeriscono l'opportunita di una pronta estensione di simili osservazioni presso le magrgiori dighe del mondo.
\end{abstract}

SUmmar. - Once again we call the attention on the interest (sometimes foundamental also for practical purposes), of the constant observation on the variations of the elastic field, in the zones where a elastic solid is submitted to continuous variations of tension, differently determined.

The twisting action, associated with the variations of tension, into a decay of the elastic modulus, testified by a more or less sensitive slowning down of the propagation velocity of the elastic waves, is translated.

The continuous observation of the elastic field in a seismic zone, joinerl with the contemporaneons observation of the variation of the apparent vertical, can be a valid help in approching the problem of the earthiuakes forecast; in the zone of Tolnezzo, for example, some seismic shocks in their preparation phase, have been surprised. In the big dans such researches, 
systematically pursuel, cau lead - as actually —, to single out a local scisinicity, natural or caused, which, with its consequences, can be fatal to the people living in the valleys below.

'The recent tragic events, happened in many places, suggest to spread rapidly such researches in the biggest dams of the world.

1. - Pii volte ormai, nella mia pluriennale esperienza sull'ambiente fisico ospitante una grande diga, ho sottolineato l'eccezionale interesse che riveste l'osservazione del campo elastico associato ai sistemi rocciosi ai quali la diga e ancorita, o, in senso pii esteso, sopportanti il bacino idrico artificiale.

Gia le prime esperienze in merito - prime in Italia e fuori - compiute dal 1949 al 1954 presso le dighe di Pieve di Cadore e del Lumiei (con particolare riguardo alla prima), riuscirono quanto mai significative. Su di esse ho riferito pii volte, in relazioni destinate alla SADE e in pubblicazioni specifiche $\left(^{1}\right)$.

Dapprima la roccia decade - ed e il decadimento più rapido e notevole - a motivo dello sbancamento, praticato con l'esplosione di cariche di esplosivo. $\Lambda$ parita di altre condizioni, il decadimento assume entita piu spiccate quanto maggiore e la carica esplosiva singolarmente usata. La causa di tale decadimento va sopratutto ricercata nel fenomeno di decompressione della roccia messa a nudo, sebbene non vada sottovalutata l'azione di minuta demolizione provocata in profondita dall'onda dirompente, legata all'esplosione.

Sono i vincoli elastici molecolari del mezzo che vengono alterati e rilassati, entro un laggio non facilmente valutabile, intorno al punto di scoppio, ben oltre la nuova superficie esterna limitante la roccia, solo in apparenza rimasta sana. Oltre che presso le dighe sopra citate, testimonianze decisive, in questo senso, sono state raccolte nella stretta del Vajont, e in Val Zoldana, presso Pontesei (sul fiume Mae, affluente del Piave) con numerosi interventi e controlli, ripetutamente eseguiti attraverso gli amni dal 1953 a1 1961 . In una delle mie piu recenti pubblicazioni, e mostrato come tale fenomeno possa essere riprodotto anche ron esperienze di laboratorio.

Ma le cause di decadimento del modulo elastico della roccia d'imposta di una diga, non si limitano a quelle legate alle esplosioni. Una volta costruito lo sbarramento, insorgono immediatamente altre cause di perturbazione; l'invaso e le sue variazioni accidentali o periodiche, l'insolazione (onda diurna) e le sue variazioni lungo l'anno solare, le variazioni della temperatura dell'aria, stagionali, periodiche e non periodiche, lente 
o rapide, ece. Anche su questi fenomeni ho potuto raccogliere una casistica quanto mai varia e probante. Nel loro rilevamento, oltre al vibrometro, si sono dimostrate particolarmente preziose le stazioni fotoclinografiche e le stazioni sismiche, operanti presso aleune grandi dighe di sbarramento (Pieve di Cadore, Tolmezzo, Vajont ...).

2. - Con riferimento alla roccia d'imposta cli una grande diga e al letto roceioso del relativo bacino idrico, puo avere influenza decisiva -ai fini del logoramento, e successiva demolizione dell'edificio roccioso la microsismicita, naturale o provocata. I)i solito, ci si limita, prima della costruzione di una grande diga, a raccogliere notizie sulla sismicita della zona prescelta. Si dimentica pero che non e tanto la sismicita normale, traducentesi in scosse telluriche piu o meno intense, che può preoccupare; le dighe, come vengono costruite oggi, possono reggere vittoriosamente all'urto dei piu violenti terremoti.

L'insidia e piuttosto legata all'eventuale esistenza di una locale attivita sismica minutissima, che sfugge alle stazioni sismiche normali, anche se strumentalmente ben dotate. Ad essa si deve la lenta, graduale, impercettibile demolizione dell'edificio roceioso, nel suo tessuto elastico. Solo una stazione sismica locale puo rivelare una così fatta microsismicita. Ed anche in questo campo, l'esperienza da me compiuta, non poteva essere piu decisiva.

La microsismicita può essere provocata. Questo è il caso verificatosi nel bacino artificiale di Pieve di Cadore. L'alterazione delle caratteristiche elastiche del mezzo, comunque provocata da una, da alcune o da tutte le cause, di cui si e fatto cenno al n. 1, non puo non tradursi in ma o piu serie di mieroscosse. Solo una stazione sismica funzionante presso la diga, puo testimoniare dell'esistenza di questa loealissima inquietudine.

ì ciò che è appunto avvenuto a Pieve di Cadore, dove fino dal 1951, la stazione sismica ivi sistemata, (Fig. 1-1a) hal dato chiarissima testimonianza di una microsismicita, nei suoi aspetti piu caratteristici, inequivocabilmente legata all'esistenza della diga e dei suoi movimenti. La Fig. 2 riproduce uno dei tanti periodi, durante i quali la crisi microsismica si è manifestata.

La microsismicita puo anche essere naturale, preesistente alla costruzione della diga, o puo essere naturalmente potenziale, riattivata pero dall'intervento umano. La piu drammatica, tragica esperienza al riguardo e stata fornita dalla stazione sismica funzionante presso la eabina comandi della diga "Carlo Semenza " del Vajont. I sismogrufi, fino dal loro primo funzionamento, sorpresero la mierosismicita in atto, in sponda sinistra, a 
monte della diga. La Fig. 3 da l'ultima fase di detta microsismicita, legata al contrasto tettonico che doveva demolire, in profondita, la roccia sostenente le pendici del monte Toc.

Ritengo superfuo insistere sul fondamentale interesse, legato alla microsismicita.

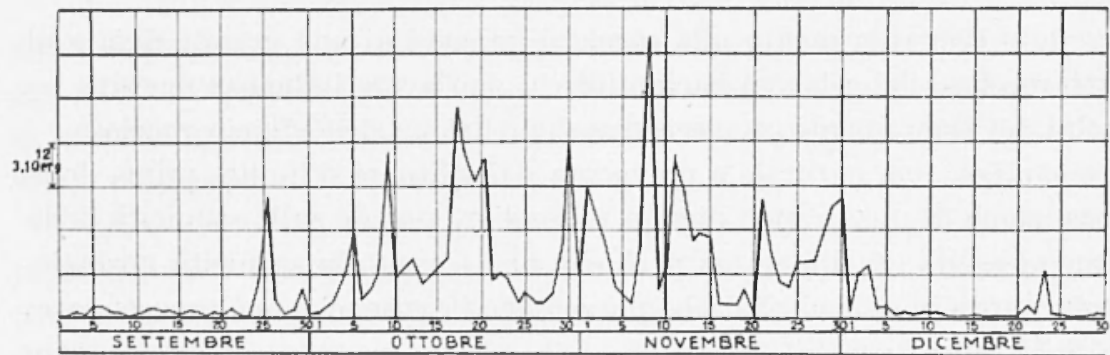

Fig. 2 - Alle notevoli flessioni verso valle osservate nell'autumno 1951 presso la diga di Pieve di Cadore, fece riscontro un'accentuata attivitit microsismica, di cui il diagramma fornisce la media energia liberata. (Nel solo 1951, presso la diga di Pieve di Cadore, forono registrate oltre 30.000 - trentamila - microscosse!)

Per quanto concerne la grande diga di Pieve di Cadore, una lieve ripresa di questo fenomeno si e avuta nella seconda meta del mese di Ottobre 1963. Essa e culminata con una scossa, nettamente avvertita dal personale della diga e nella vicina Sottocastello, avvenuta il $1^{\circ}$ Novembre. L'epicentro e risultato a solo $\mathrm{km}$ 1,8 dalla cabina comandi; lo scotimento hat avuto la sua origine a $\mathrm{km} \mathrm{1,2}$ di profondita e l'energia sviluppata e stata dell'ordine di $10^{14} \operatorname{erg}$ (Fig. $3 a, 3 b, 3 c$ ).

Sempre con riferimento a questa diga, è interessante sottolineare le conseguenze, in campo elastico, del prolungato svaso del bacino ad esso associato, dopo l'evento del Vajont. Gradatamente, dal massimo invaso dell'Ottobre 1963, il lago si e andato svuotando e, verso la fine del Marzo 1964, si poteva ritenere praticamente vuoto. Tutta la zona interessata dal lago si e trovata quindi in fase di decompressione, con conseguente alterazione della "strained region ", alterazione che si e tradotta in una seric di microscosse, avvenute nei pressi della diga dal 5 al 26 Marzo, ripetutesi nei mesi successivi e non ancora cessate. L'alterazione della risposta elastica del mezzo si e estesa però tut to intorno al bacino, dove la ridestata microsismicita e culminata in una serie di scosse, registrate durante il mese di Maggio 1964, e delle quali la Fig. 7 da alcuni epicentri (Figg. $4 a, 4 b, 4 c: 5 a, 5 b, 5 c: 6 a, 6 b, 6 c$ ). 


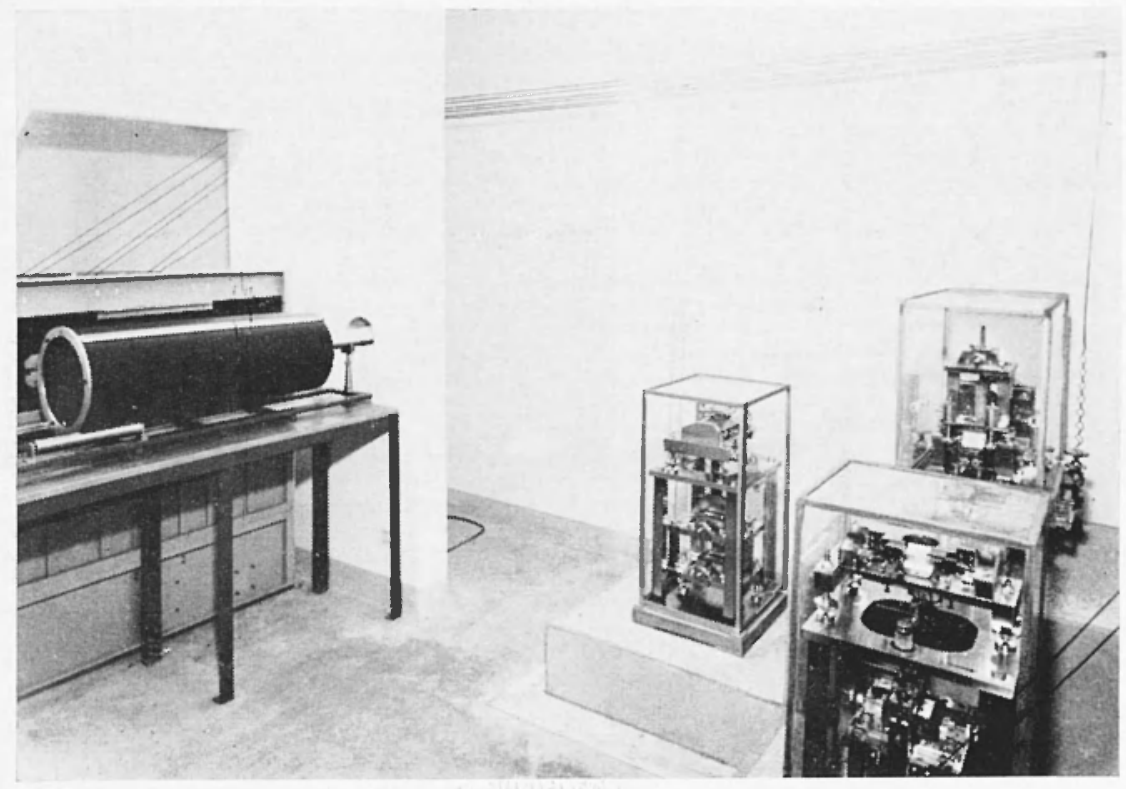

Pig. I - Stazione sismica di Pieve di Cartore, funzionante presso la locale grande diga.

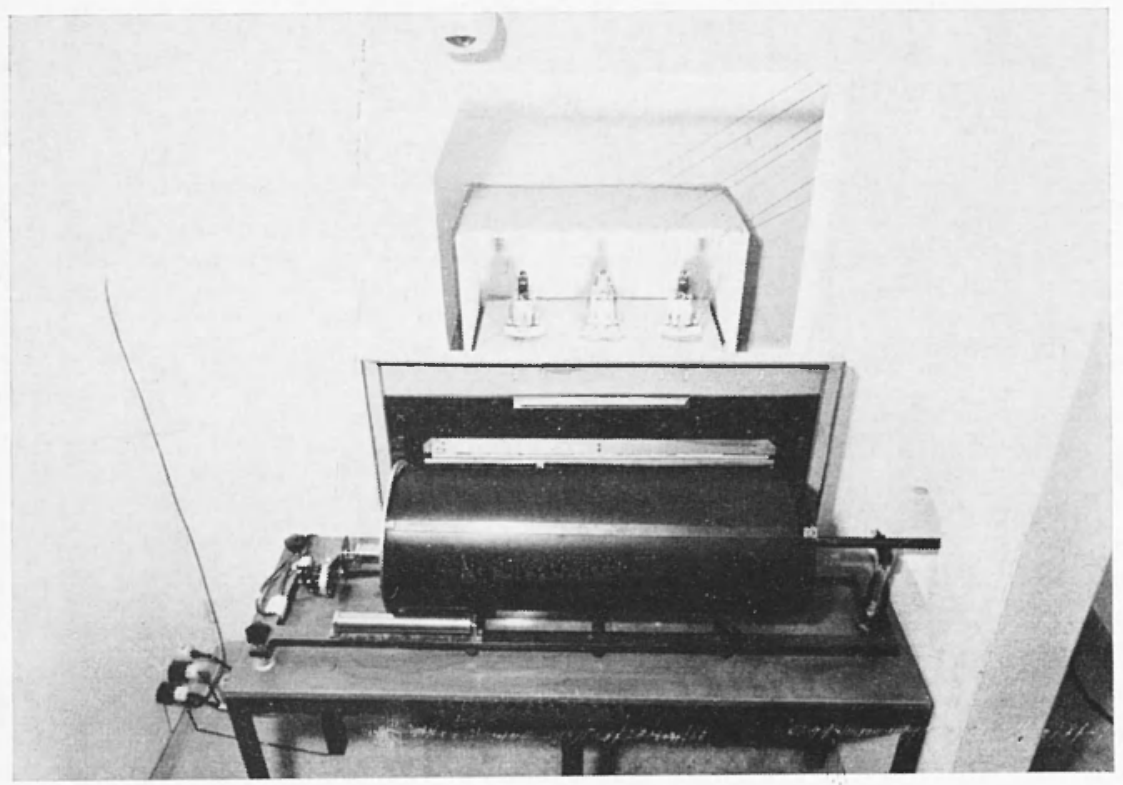

Fig. Ja - Stazione sismica di Pieve di Carlore. Particolare: registratore e galvanometri. 


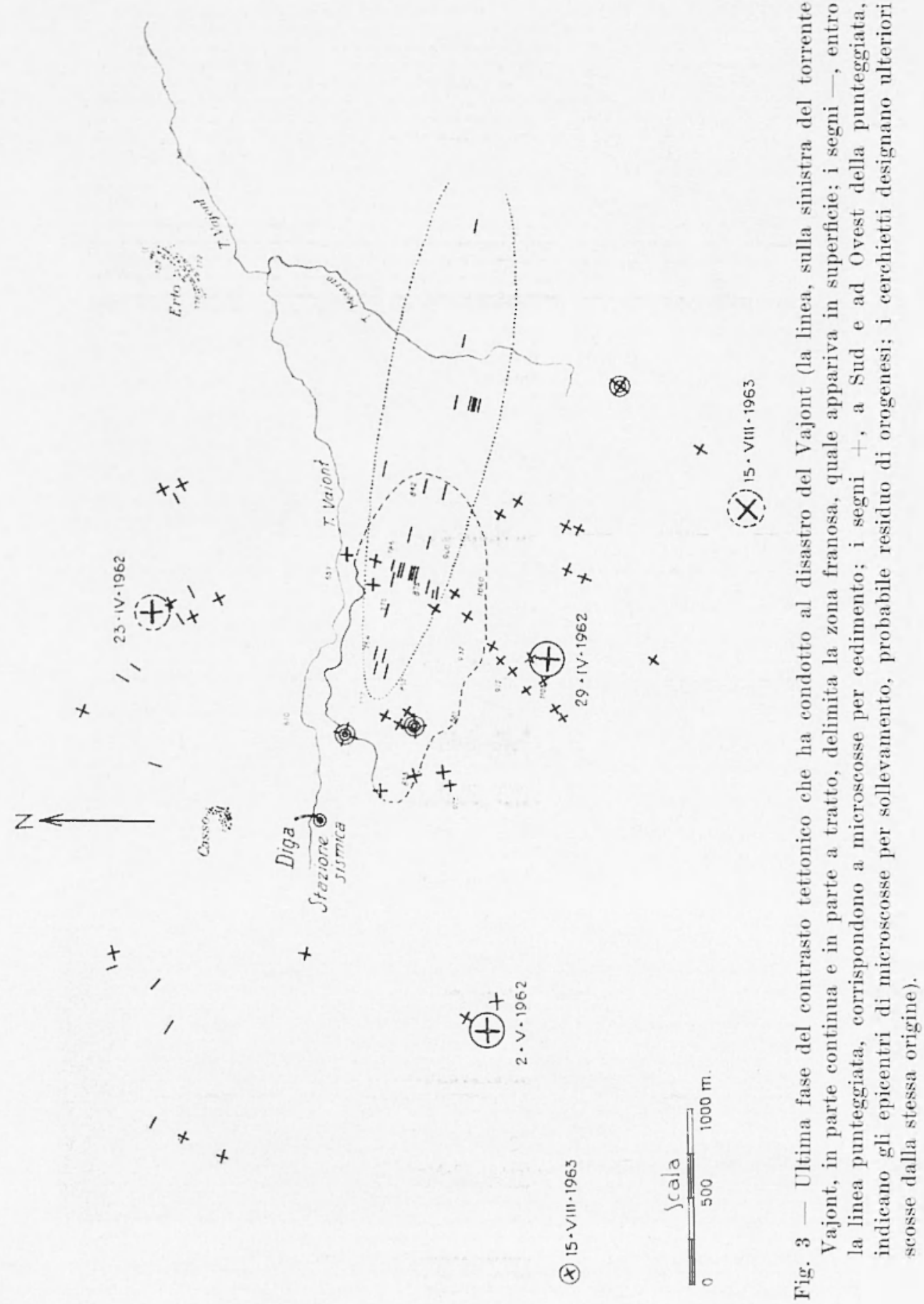




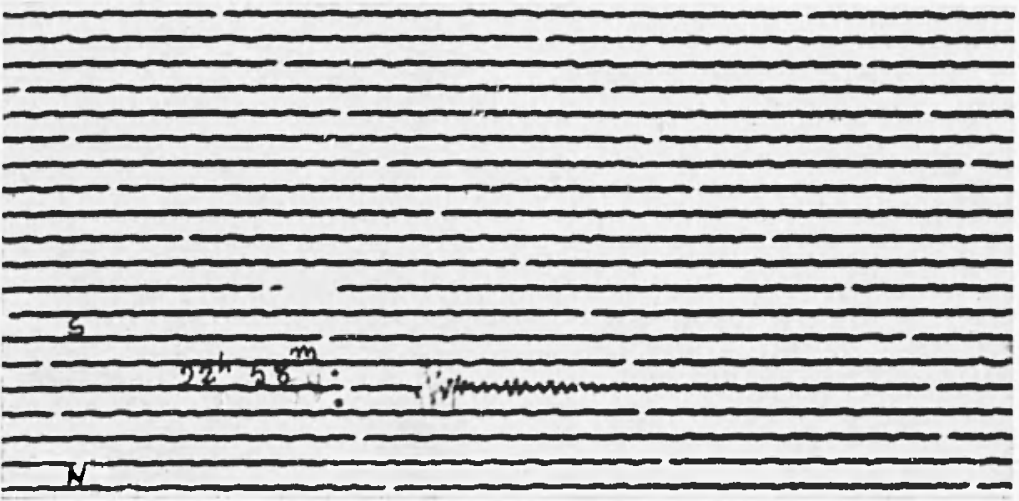

Fig. 3a - Microscossa prossima alla diga di Pieve di Cadore. 1-2-xi-1963 Componente N.S.

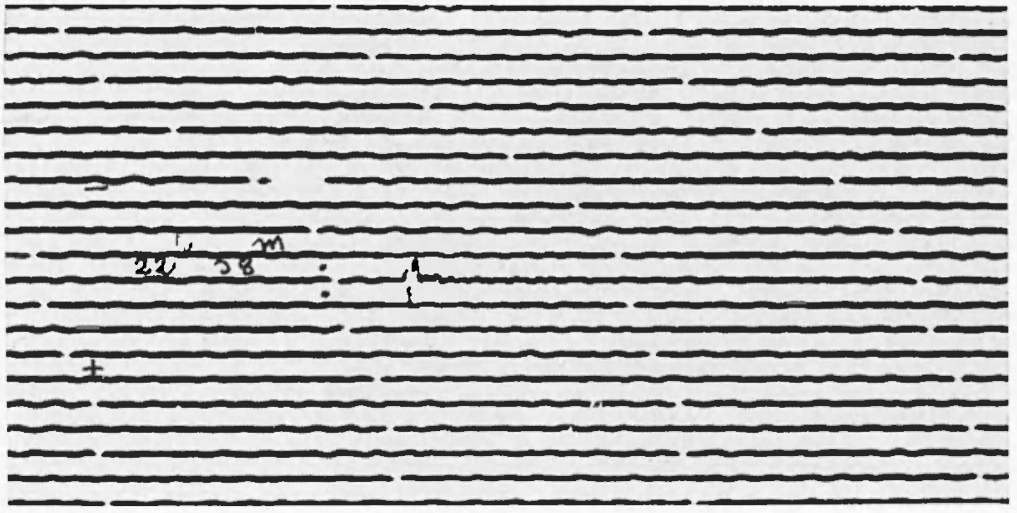
Fig. $3 b-$ Componente rerticale.

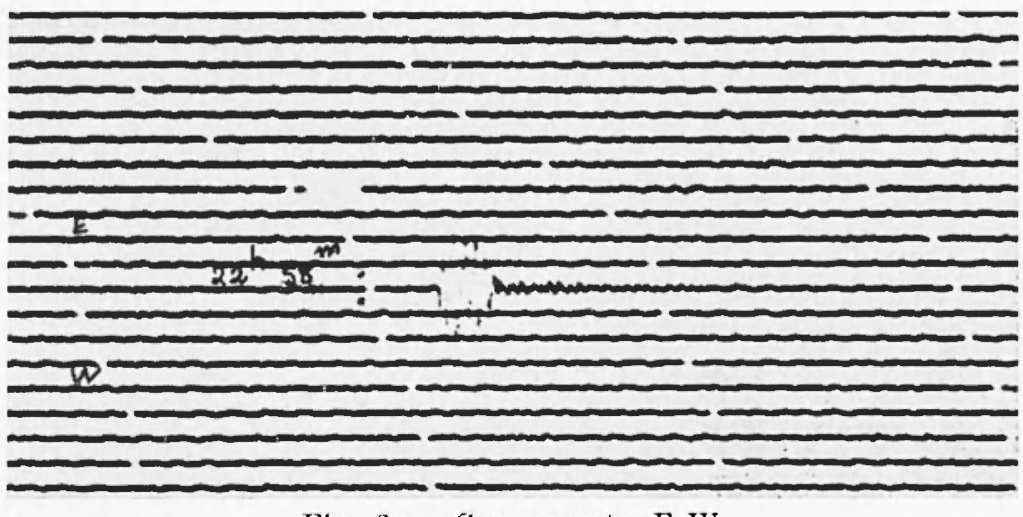
Fig. 3e - Componente E.IT. 


\begin{abstract}
$\longrightarrow \longrightarrow$
0

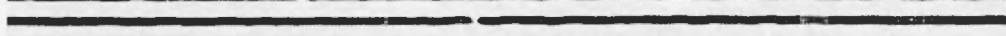
$\longrightarrow$ $\longrightarrow$ (1) 1.) -

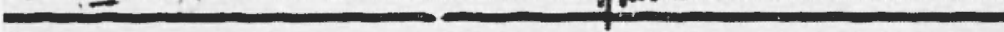
C $\longrightarrow 0$ -

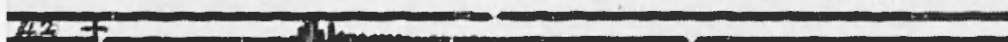
$\longrightarrow$ O 200 $\longrightarrow$ -

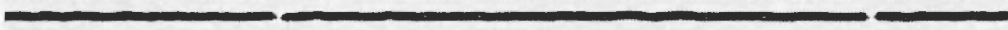

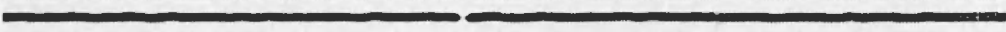
0 Fig. ta - Pieve di Carlore. Microsensse del lo-v-1964, $21^{\mathrm{h}}+2 \mathrm{~m}$ e $23^{\mathrm{h}}+5^{\mathrm{m}}$.
\end{abstract}

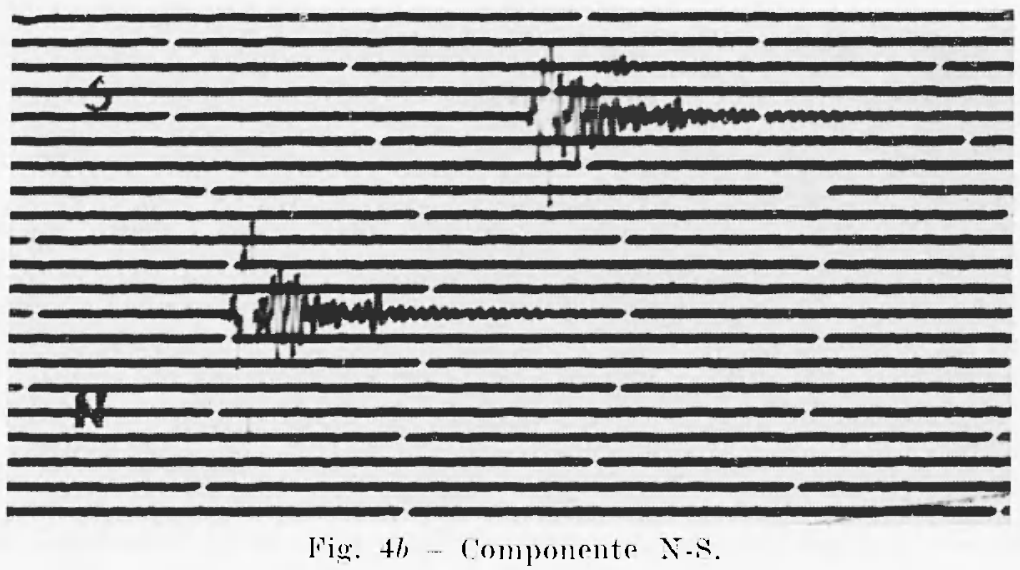
-

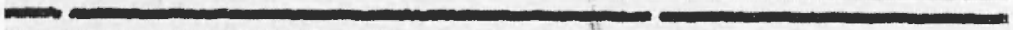

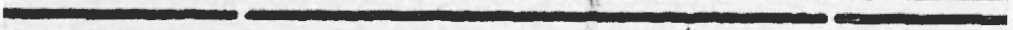

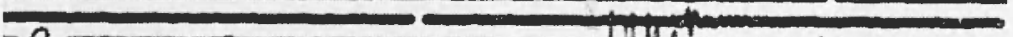

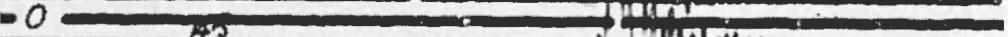

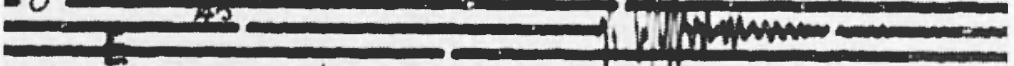

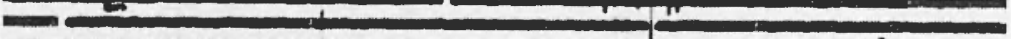

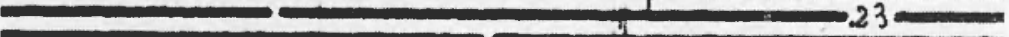
10.2

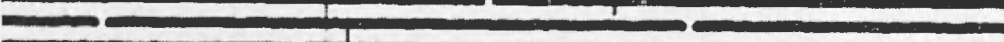

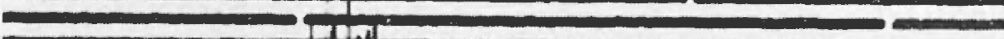
22 250 $2{ }^{25}$ -

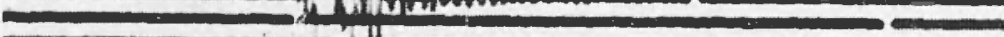
W Wh

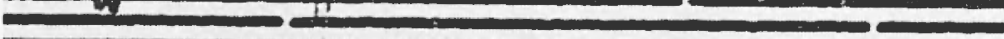

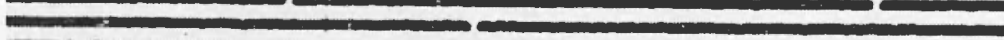

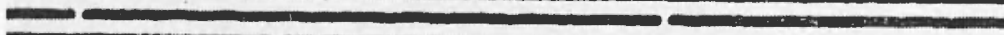

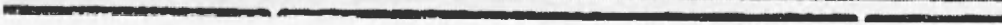

Fig. 4r: - Componente E-W. 


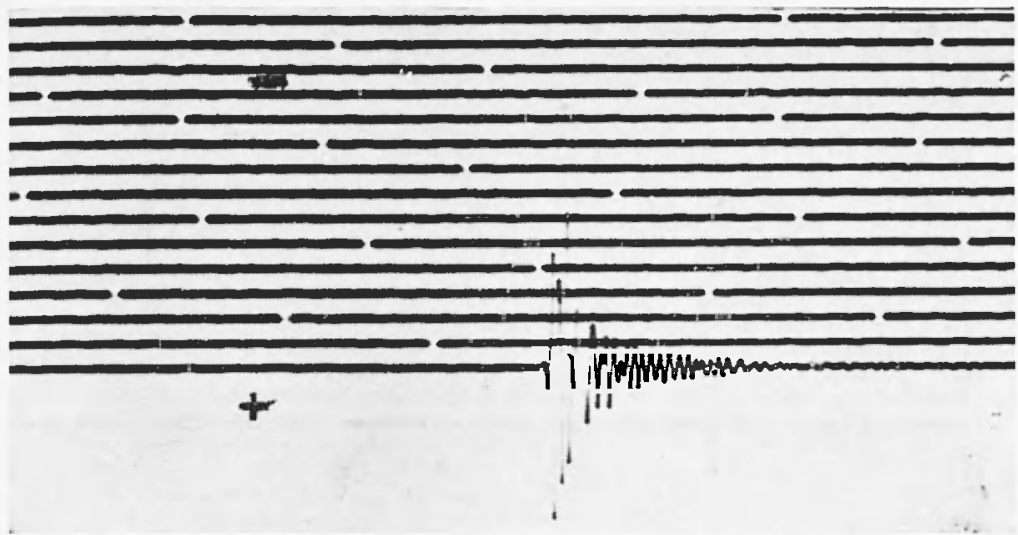

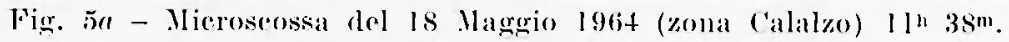
componente rerticale $\left(a \cdots 330^{\circ}: \mathrm{h}=1.2-2 \mathrm{~km} \pm\right)$.

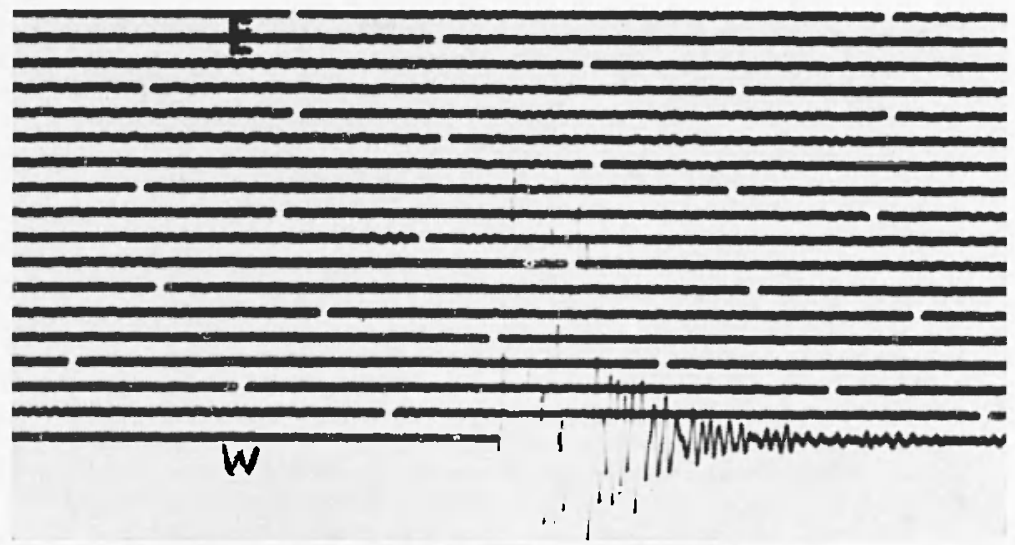

lig. $5 h$ - Componente Li.ll.

(1) 든

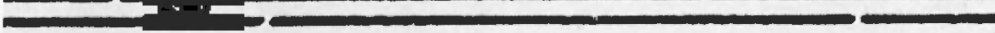

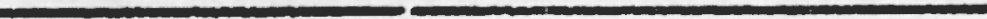

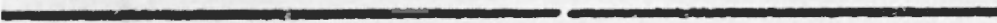
-

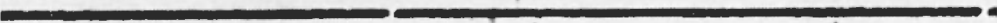
200 $\longrightarrow$ $\longrightarrow$ enom -

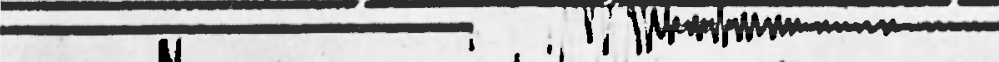

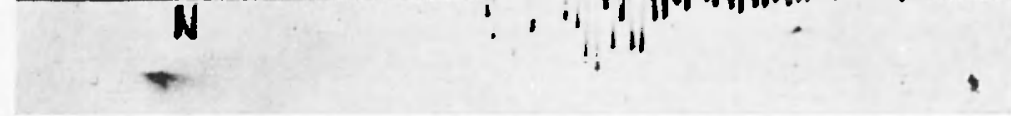

Pigr. är - Componente S-s. 


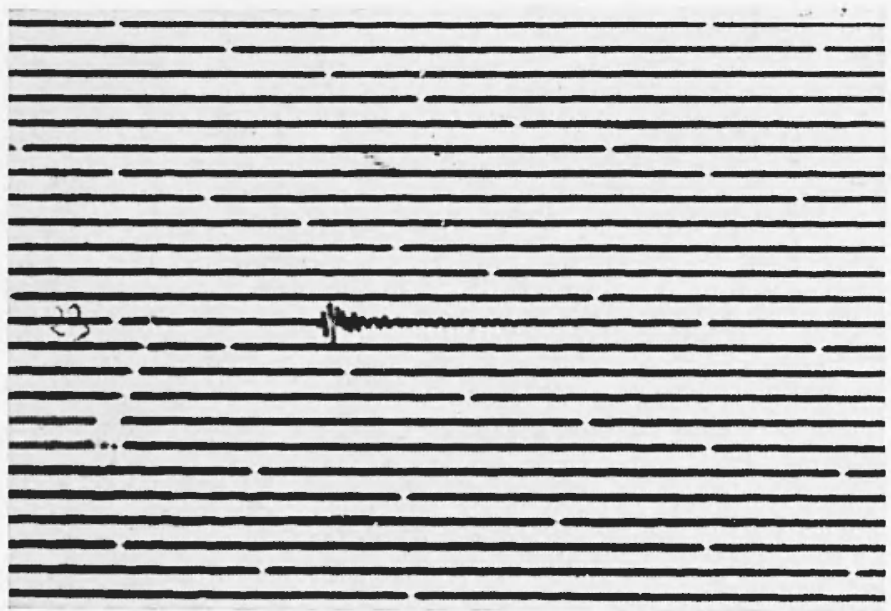

Aig. 6a - Altra microscossa rel 18-r-1964 (zona Calalzo). $19^{\mathrm{h}} 23^{\mathrm{m}}\left(\alpha-330^{\circ} ; 1_{h}-2 \mathrm{~km}\right)$.

hem 100

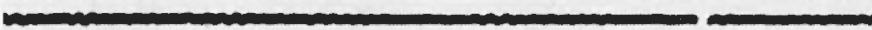
-

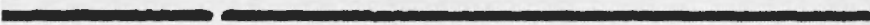

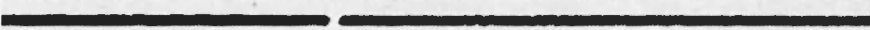

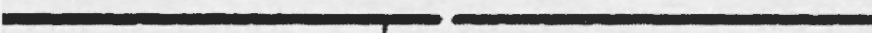
-

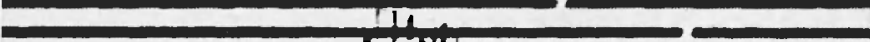

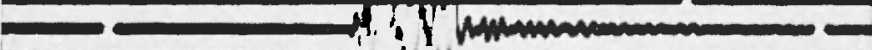

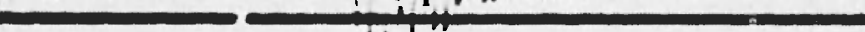
-

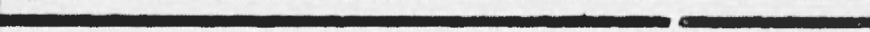
20 -

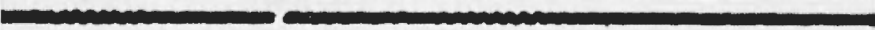

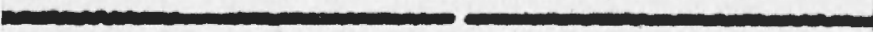

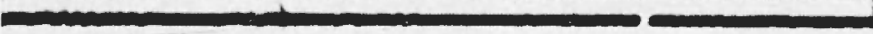

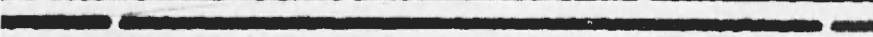
Fig. $6 b$ - Componente E.IF.

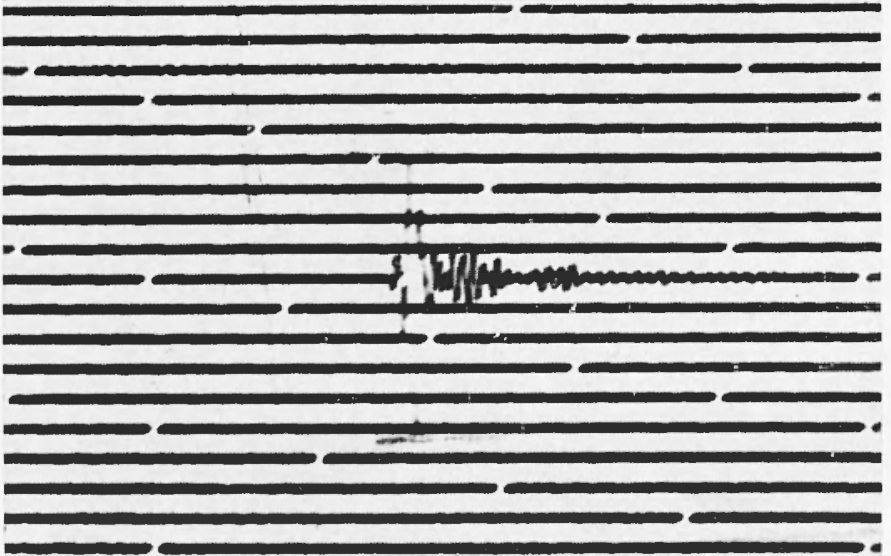
C-

$$
\text { Fig. } 6 e \text { - Componente N.S. }
$$




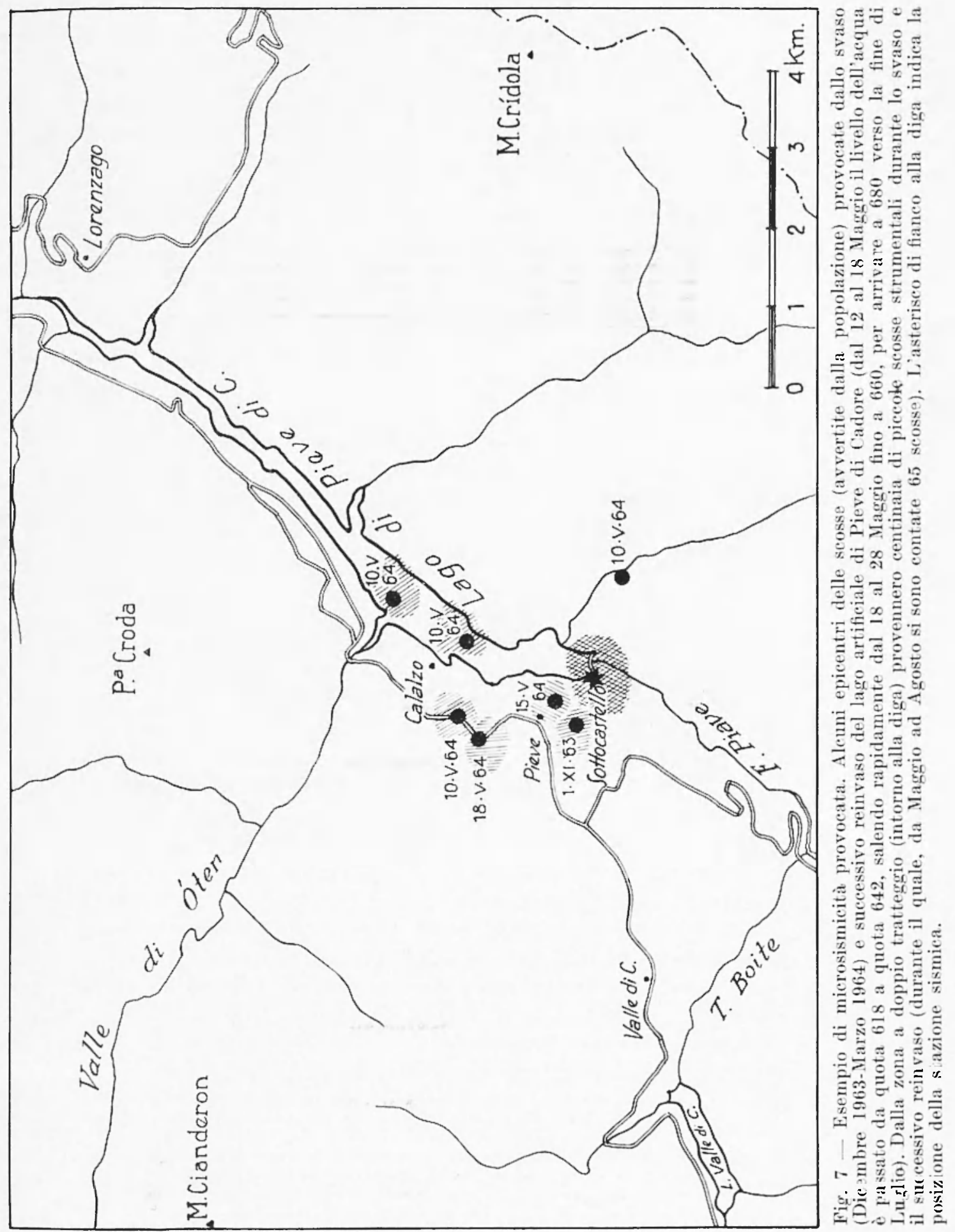


3. - L'avrento di nuove tecniche d'indagine, legata a indirizzi scientifici pin o meno diversi da quelli in precedenza seguiti, trova sempre incomprensioni ed opposizioni da parte dei sostenitori dei metodi tradizionali. L'incomprensione, la diffidenza, l'indifferenza e la sot tovalutazione non potevano certo risparmiare anche i metodi d'indagine da me introdotti nello studio dell'ambiente fisico, destinato ad ospitare una diga, e nel controllo e sorveglianza dello sbarramento, una rolta costruito. Ciò e umano.

A mio avviso, pero, alla luce - spesso drammatica - dei risultati ottenuti e dei fatti accaduti, oggi questi metodi non possono piu essere ignorati, a meno di rendersi responsabili di colpevole traseuratezza.

L'obiezione maggiore, che apertamente o tacitamente viene fatta da molti, e che la roccia non puo degradare, ahmeno in un breve volger d'amni. Ed e qui l'errore. Bisogna convincersi che il campo delle tensioni elastiche viene piu o meno profondamente modificato dall'intervento dell'uomo, dapprima in modo violento come conseguenza dello sbancamento, quindi piu lentamente ma in modo continuo, dopo la costruzione di uma grande dign.

C'e ancora chi nega la possibilità che la roccia d'imposta di una diga (la eulla del manufatto) possa flettere nelle sue caratteristiche elastiche (e quindi nella sua compattezza e resistenza). Si dimentica che i continui movimenti cui la diga viene sottoposta, periodici e non periodiei, non possono non riflettersi in eampo elastico. E non si deve credere che, tutt'al piu, tali flessioni debbano essere limitate alla roccia a contatto immediato con la diga.

Esse possono estendersi - e di fatto si estendono - nel mezzo roceioso per diecine e diecine di metri, come è appunto provato dalle esperienze che sto conducendo nell'area della diga di Pieve di Cadore dal lontano 1949. In fondo, ciò non dovrebbe meravigliare. Per dare un esempio grossolano, che presenta qualche analogia col fenomeno in questione, si pensi a ciò che si verifica quando si cammina su fine sabbia umida: tut to attomo al piede, la sabbia trascolora in un vasto alone, testimonianza del variato campo delle tensioni in un'area molto piu estesa di quella in cui la pressione si esercita. Vedansi, per citare un altro esempio, le conseguenze della pressione esereitata in una piccola zona di un pezzo di plexiglas, quale risulta dalla Fig. 8 (riprodotta in bianco e nero, dall'originale variamente colorato): anche qui, l'area in cui si verifica la variazione del campo elastico e enormemente piu estesa di quella in cui la pressione effettivamente si manifesta. 
Le enormi variazioni di pressione che la diga, nei suoi continui svariati movimenti, esercita sulla roccia alla quale e ancorata, si propagano

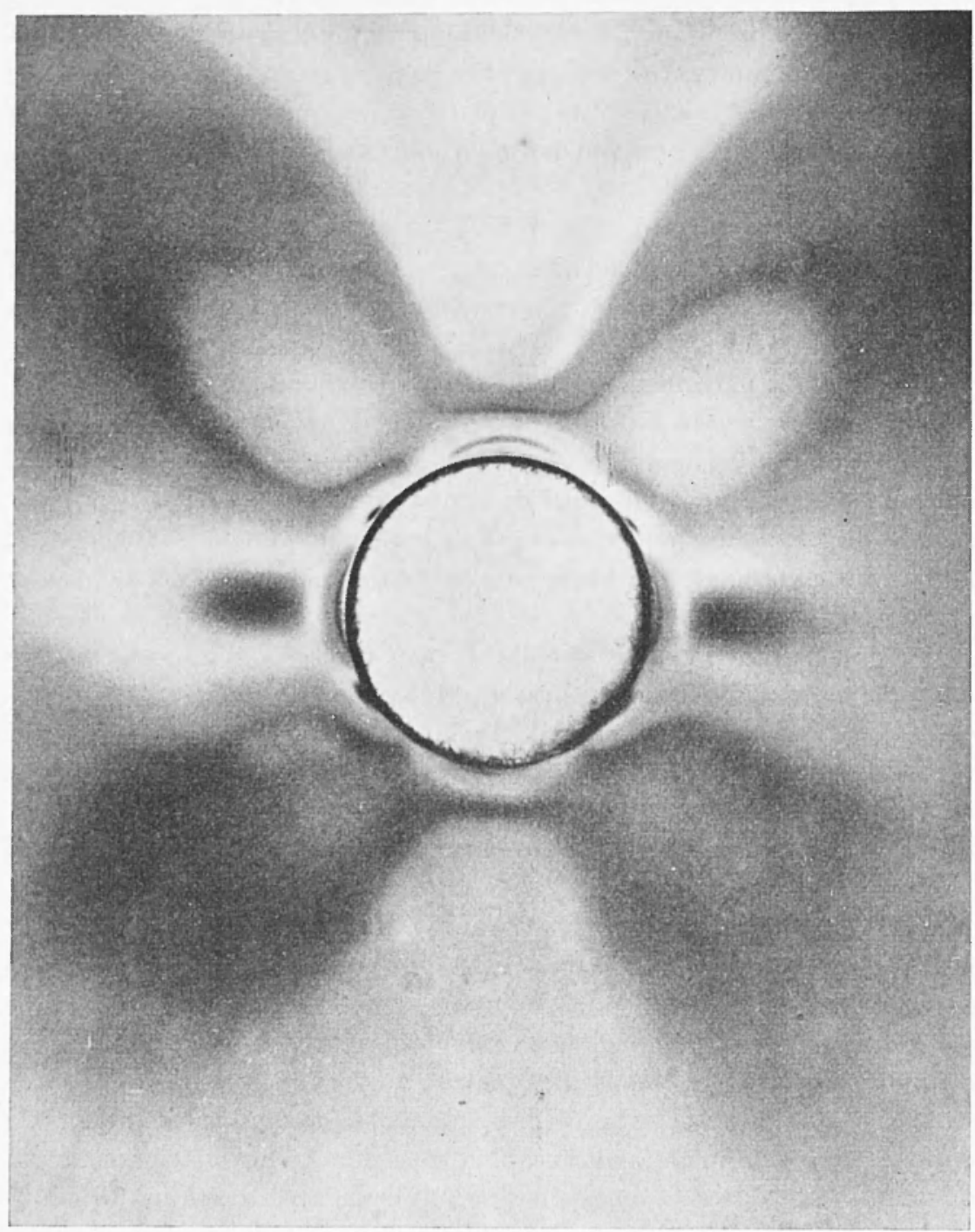

Fig. 8 - Plexiglas fortemente pressato nella zona circolare centrale.

certamente in una vasta zona tutt'intorno alla zona d'imposta, dove il campo elastico sara pertanto continuamente variato. Attesa la natura discontinua del mezzo, questo incessante lavorio - che si traduce, 
sostanzialmente, in un tormento senza soste - non puo non determinare un lentissimo, ma incessante logoramento, conseguente a formazione di minutissime lesioni, con progressivo allentamento dei legami elastici.

Che le cose stiano in questi termini e stato, del resto, chiaramente dimostrato dalle interpretazioni geofisiche della prima serie di ot to misure di triangolazioni, eseguite dalla SADE presso la diga di Pieve di Cadore, dal 1949 al 1953. E risultato, da queste analisi, che la dilatazione, la distorsione, la rotazione, la massima distorsione, e le deformazioni principali (elementi fondamentali della deformazione elastica) subiscono, da una triangolazione all'altra, variazioni talvolta notevoli, con inversione di segno: ulteriore testimonianza delle variazioni a cui e incessantemente soggetto il campo elastico in una estesa zona nei pressi della diga. I risultati di questa impegnativa indagine sono stati resi noti, oltre che alla SADE, anche nel convegno sulle grandi dighe, tenuto a New York nel $1958\left({ }^{2}\right)$.

Dopo quanto si e detto, apparirá del tutto normale che una microsismicita provocata possa insorgere intorno ad una grande diga o che una latente microsismicita naturale vi possa essere ridestata. is appunto quanto si è verificato a Pieve di Cadore e al Vajont, come venne largamente rivelato dalle locali stazioni sismiche.

4. - A conclusione di queste brevi note riassuntive, posso finalmente affermare di non essere ormai piu solo - almeno in campo internazionale - a pensare nel modo detto. Altri, in diversi Paesi, stanno orientandosi verso queste nuove vedute. Citero soltanto i Giapponesi, notoriamente all'avanguardia in questo ordine di studi. E noto ehe il modulo d'elasticita di una roccia, determinato con i metodi dinamici, presuppone la conoscenza della velocita di propagazione delle onde elastiche longitudinali e trasversali nella roccia stessa. Il decadimento del modulo elastico presuppone pertanto una più o meno sensibile diminuzione di detta velocita nel mezzo solido considerato. E quanto sto osservando nelle indagini sulle rocce d'imposta delle grandi dighe, a partire dal 1949.

Ebbene, di recente, in Giappone furono osservati mutamenti nella velocita delle onde sismiche, prima di grossi terremoti; detti mutamenti indicavano modificazioni nelle proprieta elastiche della crosta terrestre, nell'area dove il terremoto stava preparandosi. Ciò ha indotto gli esperti giapponesi ad introdurre, nel loro vasto programma di indagini tese alla previsione dei terremoti $\left({ }^{3}\right)$, anche periodiche determinazioni di velocita delle onde sismiche, provocate mediante esplosioni, in zone di elevata 
sismicita opportunamente prescelte (periodicita che, inizialmente, e prevista di un solo mese!).

Isa verita, quindi, sta facendosi strada.

5. - Un altro strumento di eccezionale utilita, prima, durante e dopo la costruzione di una grande diga, si e rivelato il fotoclinografo. Mi limiteró a dire di esso poche parole, anche se il suo uso debba ormai ritenersi indispensabile, ai fini della determinazione dei fondamentali lineamenti

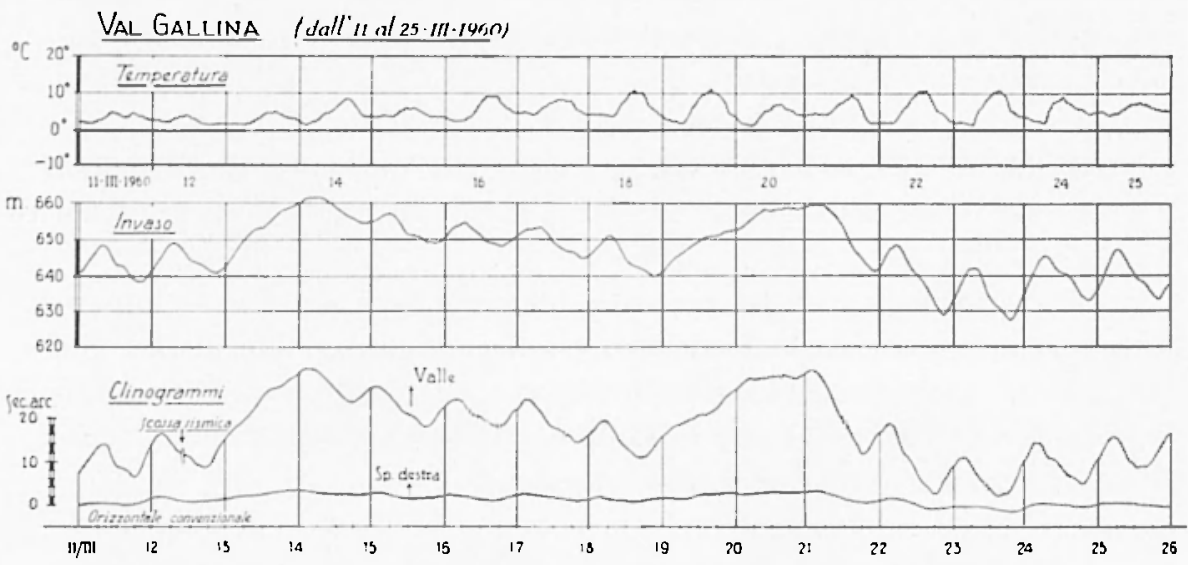

Fig. 9 - Alla base dei conci, la diga flette quasi esclusivamente sotto l'azione dell'invaso, come nell'esempio qui riportato, in cui - come di consuetudine - la componente valle monte segue fedelmente le variazioni di livello dell'acqua nel bacino idrico.

dinamici di una localita destinata ad ospitare una diga o - una volta costruita quest'ultima - a controllarme il comportamento nel tempo. Come prontezza, sensibilita, fedelta e continuita di risposte nessun apparecchio può rivaleggiare con il fotoclinografo, specie nella versione da me introdotta, da oltre 15 amni, nello studio - in senso lato - dei grandi sbarramenti. Su di esso, e sui risultati del suo uso, ho pii volte riferito; e qui non intendo ripetermi. Per quanto riguarda la sensibilita e la fedelta alle variazioni di pressione sopra uno sbarramento, legate alle variazioni dell'invaso (l'acqua del bacino artificiale creato dalla costruzione della (liga), bastera l'esempio fornito dalla Fig. 9 .

Non meno interessanti risultano le registrazioni connesse con l'onda diuma (provocata dall'insolazione diurna e dalla successiva scomparsa del sole); con le variazioni di temperatura dell'aria, specie quelle stagionali, ece. 


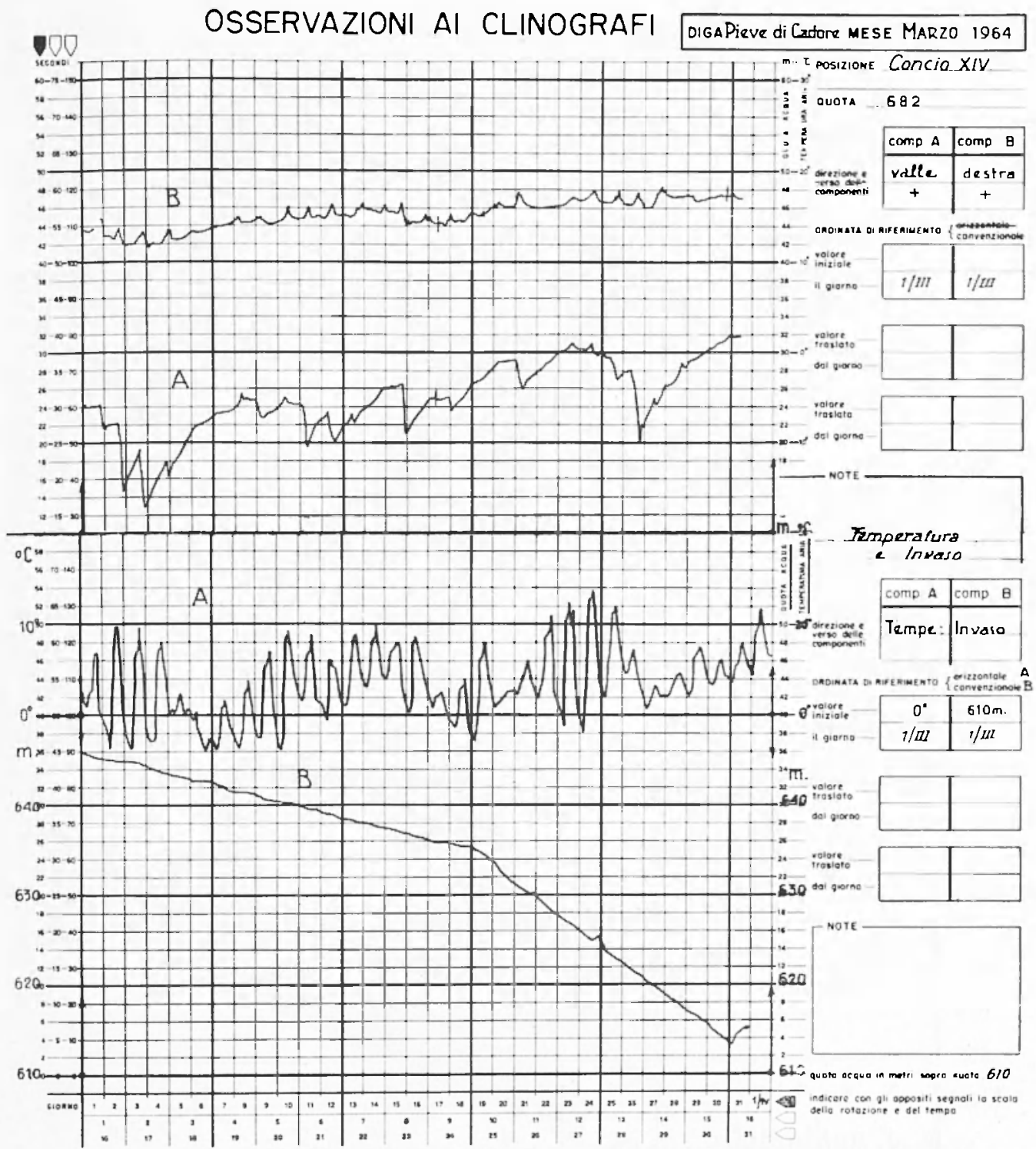

Fig. 10.

A questo riguardo, va ricordato che l'onda diurna e praticamente legata all'esistenza del lago a monte della diga e nase appunto dal contrasto fra la variazione diurna della temperatura della parete a valle (sopra tutto per effetto dell'insolazione) a la pressoche costante tempera- 


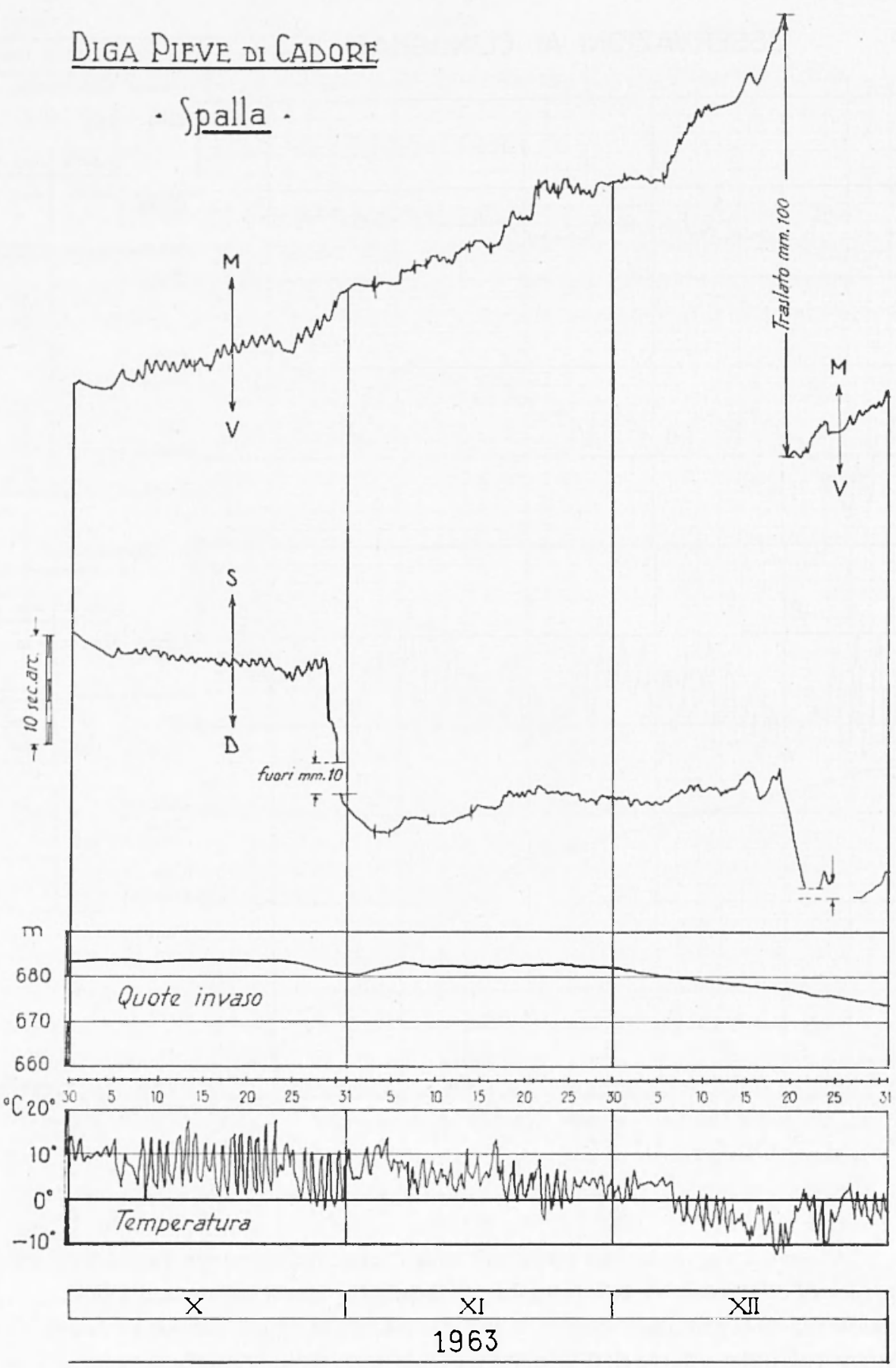

Fir. 11 -Postazione clinografica in roccia, sponda destra, a contatto con la diga. All'improvviso raffreddamento dell'aria, verificatosi verso la fine di Ottobre - e ripetutosi verso la meta di Dicembre - (1963), fa riscontro un micro-cedimento della roccia, rivelato dalla componente clinografica tangenziale alla valle (SD), Iungo la quale massima si manifesta la pressione della diga, nella sua flessione verso valle. 


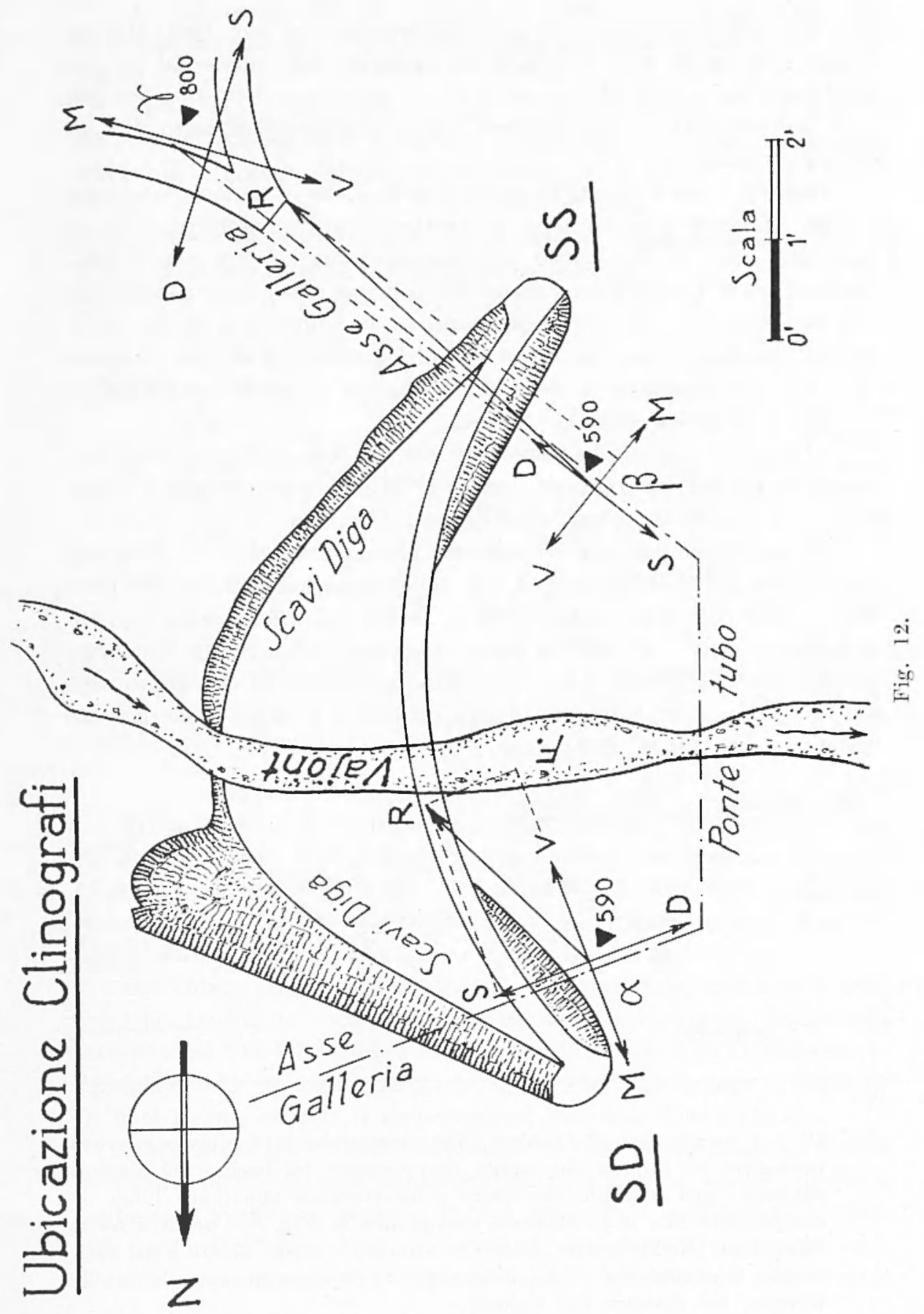


tura della parete a monte, sommersa nelle acque del lago. Ogni diga ha esempi cospicui di onda diuma, la cui ampiezza diminuisce dal coronamento alla base della diga e varia altresi al variare delle stagioni (nei mesi invernali, di fatti, si attenua, fino a scomparire). Questo, in condizioni normali.

Quando, come e avvenuto per la diga di Pieve di Carlore (v. quanto e stato osservato al n. 2), tutta la struttura resta all'asciutto, allora il comportamento del manufatto alle variazioni termiche viene profondamente alterato. L'onda diurna pressoche scompare e, per quanto si riferisce alle variazioni piu lente di temperatura, ad un amento di questa corrisponde una flessione a valle, e ad una diminuzione un ritorno a monte (Fig. 10): giust'appunto il contrario di quanto si verifica in condizioni normali (con bacino pieno).

I'uso del clinografo ha provato ${ }^{(1)}$ che, con il passar degli amni, il calcestruzzo subisce un graluale, notevole irrigidimento, mentre la roccia d'imposta decade in campo elastico, ece. (Fig. 11).

Ia anche in posizioni discoste da uno sbarramento, il clinografo può fornire preziose indicazioni sul comportamento del sistema roccioso, eni la diga e ancorata (v. per es. la Fig. 12). Come dalla Fig. 13, la flessione verso valle subita dalla componente clinograflea, oscillante secondo l'asse della valle, e stata ottenuta in occasione di ma improvvisa alluvione che ha bruscamente invasato il bacino a monte della diga del Vajont a circa $60 \mathrm{~m}$. dalla diga.

\section{Diga del Vajont - Spalla sinistra}

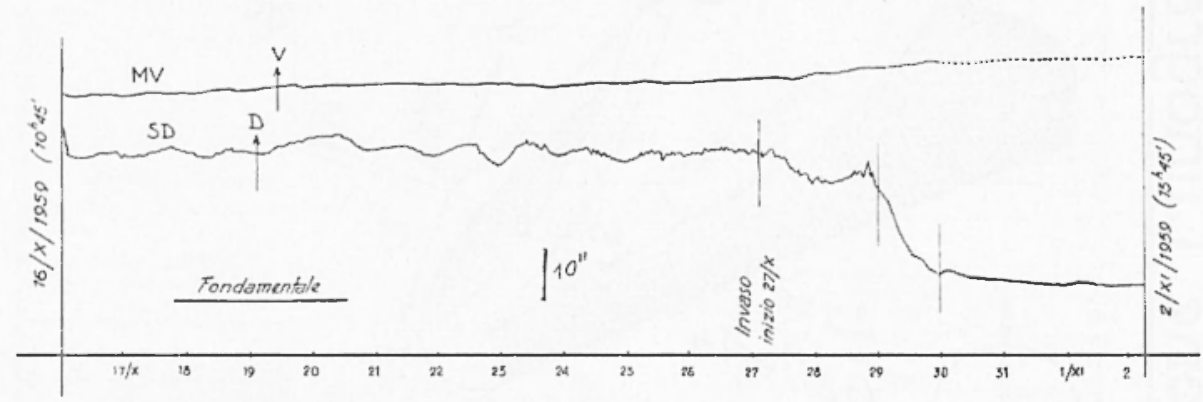

Tig. 13 - A partire dal 27 Ottolore 1959, una prolungata alluvione aveva provocato un improvviso rapido riempinento del bacino del Vajont, causando una sensibile variazione della verticale apparente lungo la componente SD, nella stazione clinografica $B$ (Fig. 12) posta a $55 \mathrm{~m}$ dalla diga. [Nell'originale, la scritta "invaso - inizio $27 / X$ » ̀̀ del compianto Giovanni De Vido, tragicamente scomparso, con il fratello Giorgio, nel disastro del Vajont]. 
Il che significa che il complesso di roccia di ancoraggio della diga ebbe a subire una flessione, ancora sensibile, per uno spessore di ca. 60 metri.

Tutto ciò a riprova di quanto e stato detto nel n. 4, circal la notevole estensione delle variazioni delle tensioni elastiche, destate nella roccia dalle flessioni di uno sbarramento.

6. - Del resto l'uso del fotoclinografo su larga scala puo portare a ben altre acquisizioni in campo seientifico.

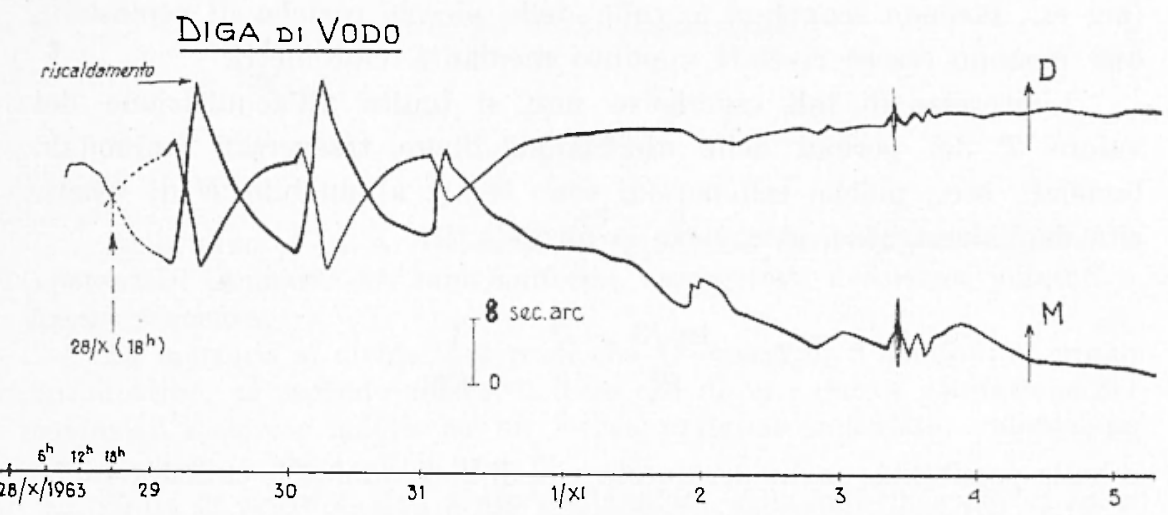

Fig. 14.

L'osservazione continua delle variazioni della verticale apparente, sia pure a piccolo ingrandimento, puo scrvire a sorprendere fenomeni geolisici, di carattere piu vasto, comunque legati all'attivitit della crosta terrestre. Cio es stato gia messo in evidenza in particolari pubblicazioni ${ }^{4}$ ). Qui mi limito a riferire un semplice esempio dell'utilita di tali osservazioni, we andrebbero largamente diffuse sul suolo nazionale, specie nolle zone sismiche. La Fig. 14 riproduce un tratto della registrazione clinograffica, ottenuta dal 3 al 4 Novembre 1963, presso la piccola diga di Vodo (sot to l'Antelas, nelle Dolomiti del Cadore, fra Pieve di Cadore e Cortina). In quei giomi, essendo il cielo coperto, mancerva l'ondal diurnal.

Sella not te fra il 3 e il 4 Novembre, si ebbe la registrazione di una variazione della verticale apparente in tutta la zona, corrispondente a oscillazioni aventi un periodo di $2^{\text {h }}, 5$ circa. Tale oscillazione e risultata pii netta sulla comp. valle monte (ampiezzal 1 sec. d'arco (a.). $\mathrm{E}$ interessante notare che, durante questo "scricchiolio " della crosta, 
e stato registrato anche un lievissimo terremoto (vedasi vibrazione nel corso dell'oscillazione accennata). Tutto cio, naturalmente, i passato inosservato alla popolazione.

7. - Con particolare riferimento alle dighe, l'uso del vibrometro può risultare di grande interesse non solo per la determinazione diretta delle caratteristiche elastiche del calcestruzzo, e della loro variazione nel tempo, ma anche per il rilevamento della risposta elastica dei conci delle dighe stesse, come elementi vibranti. Ogni concio ha periodi propri di oscillazione libera, come i rebbi di un diapason. Opportunamente eccitati (per es., facendo scoppiare a valle delle piccole cariche di esplosivo), essi possono essere rivelati appunto mediante vibrometri.

L'interesse di tali esperienze non si limita all'acquisizione del valore $T$ dei periodi delle oscillazioni libere trasversali uninodali, binodali, ecc.; poiche tali periodi sono legati al modulo $E$ di elasticità del calcestruzzo, attraverso la formula $\left({ }^{4}\right)$

$$
T=\frac{4 \pi \sqrt{3}}{\vartheta^{2}} \cdot \frac{l^{2}}{b} \cdot \frac{1}{\sqrt{E / \varrho}},
$$

si ha la possibilita, anche per questa via, di determinare le earatteristiche elastiche del mezzo sollecitato. Cio permette di seg'uire, nel tempo, l'eventuale mutare di tali caratteristiche, che sara rivelato dal mutare dei su detti periodi di oscillazione libera dei conci.

\section{BIBLIOGRAFIA}

(1) Calor P., Aspetti della dinamica di rocee, calcestruzzo ed acque. "Annali di Geofisica ", XV, 2-3, 1962. (Contiene, fra l'altro, un'ampia bibliografia sui lavori da me pubblicati sull'argomento, a partire dal 1950).

(2) Calor P., SpadFa M. C., Geophysical Interpretations of the first Series of Triangulations carried out at the Pieve di Cadore Dam. VI Congres des Grands Barrages, New York, 1958.

(3) Chuji Tsubor, Kiyoo Wadati, Takammo Itagrwara, Prediction of Earthqualies (Progress to Date and Plans for Further Development). Earthquake Research Institute, University of Tokyo, 1962.

(') Caror P., La geofisica e le grandi dighe. "I'Energia Elettrica ", XXXIX, 1,1962 . 